\title{
A propos de la teneur des laits individuels et de mélange en matières minérales et urée
}

(fin)

par

H. MAHIEU*, F. M. LUQUET** et L. MOUILLET**

Collaboration Technique A. NORMAND

TROISIÈME PARTIE

\section{OLIGOELEMENTS ET NUTRITION}

I. RESUME BIBLIOGRAPHIQUE

1.1. Oligoéléments et sols.

1.2. Oligoéléments et fourrages.

1.3. Oligoéléments et ruminants

1.3.1 Le cuivre - 1.3.2. Le manganèse - 1.3.3. Le zinc - 1.3.4. Relations entres éléments - 1.3.5. Carences.

1.4. Oligoéléments et l'homme

1.4.1. Le fer - 1.4.2. Le cuivre - 1.4.3. Le zinc - 1.4.4. Le manganèse - 1.4.5. Le magnésium - 1.4.6. Le plomb - 1.4.7. L'arsenic 1.4.8. Le mercure - 1.4.9. Le plomb.

1.5. Conclusion.

II. RESULTATS D'ENQUETES (1)

2.1. Eléments de pollution.

2.2. Oligoéléments.

2.3. Essai sur les laits de troupeaux (1973) selon les races et les régions.

Travail effectué dans le cadre des études et enquêtes financées par l'I.T.E.B. (période 1973 - 1974 - 1975).

* I.T.E.B., 149, rue de Bercy - 75579 Paris cedex 12.

** Labcodral, 13, rue de l'Université - 59509 Douai.

(1) Le résumé bibliographique est tiré du compte rendu du dernier Congrès sur les "Oligoéléments et la Nutrition ". Les résultats d'enquêtes concernent des résultats de l'enquête 1973 classées en fonction des races et des régions. 


\section{Résumé bibliographique}

L'existence d'aires géographiques de carence en oligoéléments ou de " régions à endémies » prouve que la carence est plus ou moins liée à la nature du sol. Allaway a établi un cycle des éléments minéraux (fig. 3).

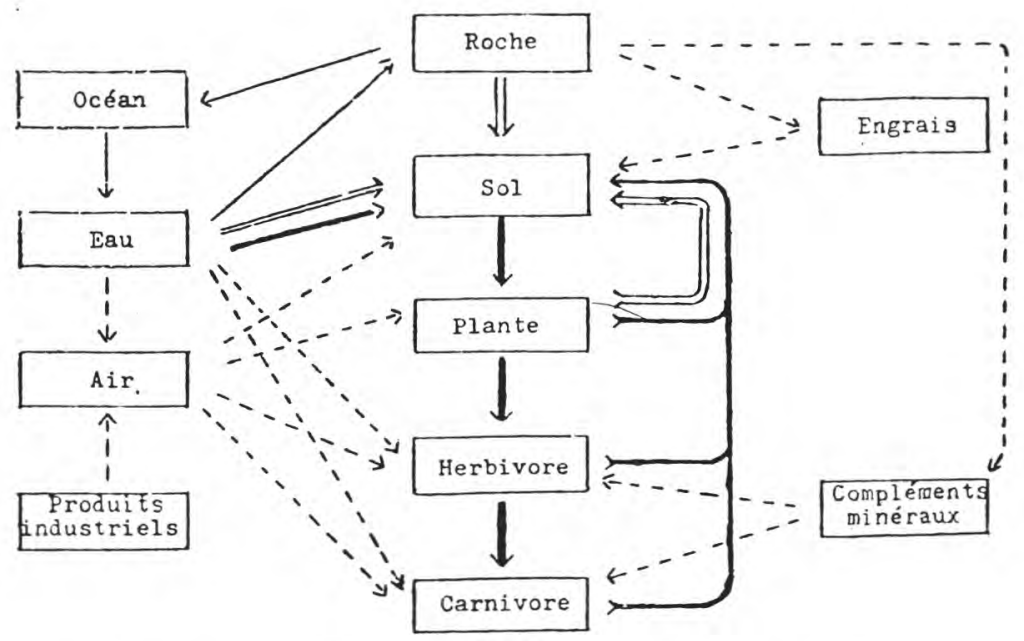

fig. 3

Cycle des éléments minéraux (schéma simplifié tiré de Allaway)

Processus à l'échelle du milliard d'années (géologie).

Processus à l'échelle de la centaine d'années (pédologie).

Processus à l'échelle de quelques années (cultures).

Ce transfert complexe des minéraux de la roche à l'animal obéit à chaque niveau à certaines lois. Mais ces lois sont-elles suffisamment connues pour permettre de prévoir, donc de prévenir, une carence ?

\subsection{Oligoéléments et sols}

La figure 4 présente le processus mis en jeu au cours de l'altération des roches.

Ce processus donne naissance à des formes plus ou moins mobiles, ou bloquées pour chaque élément. Ces formes sont les résultats de réactions simultanées plus ou moins compétitives, spécifiques de cet élément dans les conditions de milieu du moment. Les variations de conditions de milieu sont naturelles, mais le plus souvent induites par l'homme (traitements divers en agriculture, rejets de polluants...). 


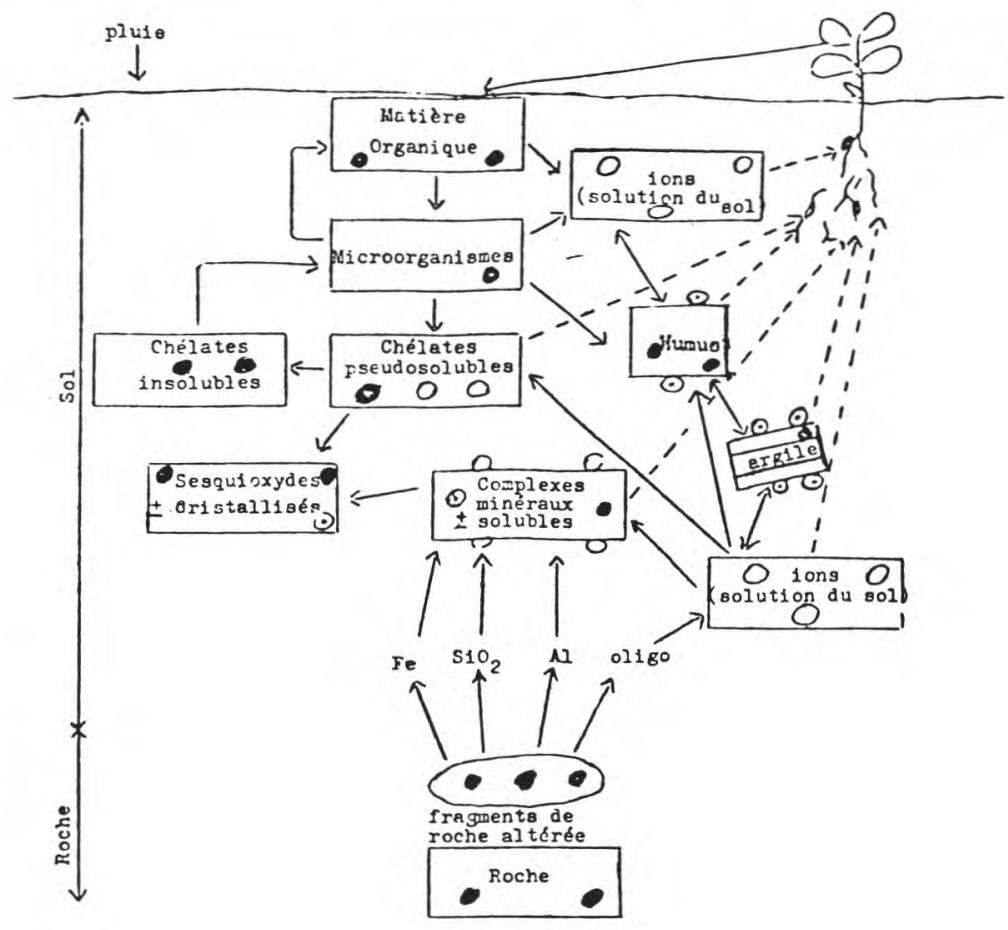

\footnotetext{
0 élément soluble, très accessible, et très woblle

$\odot$ élésent adsorbé, assez accesible, fonction du $\mathrm{pH}$

- élézent bloqué, 1naccessible, non moblle
}

fig. 4

Dynamique des oligoéléments dans less sols (schéma simplifié tiré de Dautria)

Ces variations modifient d'autant plus les équilibres en jeu que les propriétés physico-chimiques de l'élément varient avec le facteur en cause.

Ainsi pour le cuivre, le manganèse et le zinc, les formes et les teneurs sont indiquées dans le tableau 60.

Sont reportées dans le tableau 61 les teneurs en oligoéléments de certaines roches mères et dans le tableau 62 lés' différents sols pour lesquels une carence ou une toxicité ont pu être observées en ce qui concerne le cuivre, le manganèse et le zinc.

Les caractéristiques pédologiques des principaux types de sols sont rapportées dans le tableau 63. 
TABLEAU 60. - Cuivre, manganèse, zinc. Forme et teneur dans les sols (en ppm)

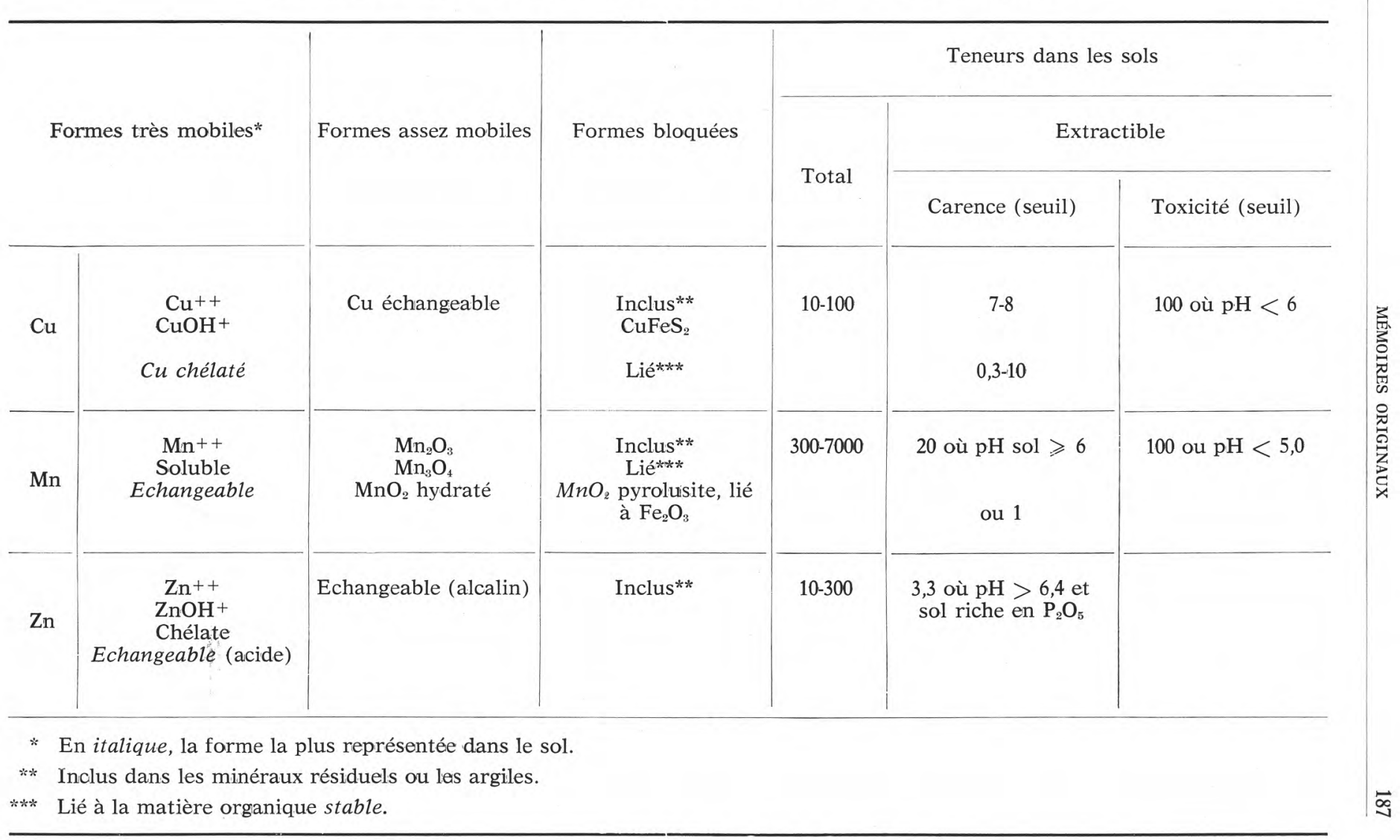




\begin{tabular}{|c|c|c|c|c|c|c|c|c|c|c|}
\hline \multirow{2}{*}{ Roche } & \multicolumn{10}{|c|}{ Eléments } \\
\hline & $\mathrm{Fe}^{*}$ & $\mathrm{Mn}^{*}$ & $\mathrm{Ni}$ & $\mathrm{Cu}$ & Co & $\mathrm{Zn}$ & Mo & B & I & $\mathrm{Se}$ \\
\hline $\begin{array}{l}\text { Croûte terre } \\
\text { (moyenne) }\end{array}$ & 50000 & 1000 & 100 & 70 & 40 & 80 & 2,3 & 10 & 0,3 & 0,09 \\
\hline \multicolumn{11}{|l|}{ Roches éruptives**: } \\
\hline $\begin{array}{l}\text { R. ultrabasiques } \\
\text { Basaltes } \\
\text { Granodiorites } \\
\text { Granites*** }\end{array}$ & $\begin{array}{c}95000 \\
85000 \\
27000 \\
29 \text { à } 14000\end{array}$ & $\begin{array}{c}1500 \\
2000 \\
600 \\
540-390\end{array}$ & $\begin{array}{r}2000 \\
160 \\
8 \\
15-4\end{array}$ & $\begin{array}{c}20 \\
87-100 \\
35 \\
30-10\end{array}$ & $\begin{array}{r}200 \\
48 \\
10 \\
7-1\end{array}$ & $\begin{array}{c}30 \\
105-130 \\
60 \\
60-40\end{array}$ & $\begin{array}{l}0,2 \\
1,4 \\
1 \\
1-4\end{array}$ & $\begin{array}{l}1,0 \\
5 \\
15 \\
15\end{array}$ & $\begin{array}{l}0,01 \\
0,5 \\
0,4 \\
0,5\end{array}$ & $\begin{array}{l}0,05 \\
0,05 \\
0,05 \\
0,05\end{array}$ \\
\hline \multicolumn{11}{|c|}{ Roches sédimentaires: } \\
\hline $\begin{array}{l}\text { Argiles } \\
\text { Schistes } \\
\text { Grès } \\
\text { Calcaires }\end{array}$ & $\begin{array}{r}33000 \\
47000 \\
9800 \\
3800\end{array}$ & $\begin{array}{c}670 \\
850 \\
? \\
1100\end{array}$ & $\begin{array}{r}95 \\
68 \\
2 \\
20\end{array}$ & $\begin{array}{c}57 \\
45 \\
10 \\
4-20\end{array}$ & $\begin{array}{l}20 \\
10 \\
0,3 \\
0,1\end{array}$ & $\begin{array}{l}80 \\
95 \\
16 \\
20\end{array}$ & $\begin{array}{l}2 \\
2,6 \\
0,2 \\
0,4\end{array}$ & $\begin{array}{l}3-300 \\
100 \\
35 \\
20\end{array}$ & $\begin{array}{l}1 \\
2,2 \\
1,7 \\
1,2\end{array}$ & $\begin{array}{l}0,6 \\
0,6 \\
0,05 \\
0,08\end{array}$ \\
\hline
\end{tabular}

* Le fer et le manganèse ne sont pas des « oligoéléments » dans les roches mais sont donnés à titre de comparaison pour les oligoéléments qui les accompagnent.

** Les roches éruptives sont classées par ordre de basicité décroissante dans ce tableau.

*** Les deux chiffres correspondent aux deux séries extrêmes de granites ; le premier, aux calcosodiques, les seconds aux potassiques (plus « acides"). 
TABLEAU 62. - Cuivre, zinc, manganèse dans les sols (d'après Lamand et Périgaud)

\begin{tabular}{|c|c|c|c|c|}
\hline & \multirow{2}{*}{ Sols carencés* } & \multirow{2}{*}{ Sols toxiques* } & \multicolumn{2}{|c|}{ Analyses** } \\
\hline & & & $\begin{array}{c}\text { Méthode } \\
\text { d'extraction }\end{array}$ & $\begin{array}{l}\text { Seuil de carence } \\
\mathrm{mg} / \mathrm{kg} \text { de terre }\end{array}$ \\
\hline Cuivre & $\begin{array}{l}\text { Acides : granites blanas, grès, graviers, podzols, tourbes } \\
\text { acides. } \\
\text { Neutres : balsates très lessivés, andésites peu altérables. } \\
\text { Calcaires : certains sols crétacés, dunes calcaires. }\end{array}$ & Anciennes vignes. & $\begin{array}{l}\text { Total } \\
\text { (nitroperchlorique) }\end{array}$ & 7 à 8 \\
\hline Zinc & $\begin{array}{l}\text { Acides graveleux (pauvres en zinc). } \\
\text { A pH élevé, blocage par calcium. } \\
\text { Neutres, riches en } \mathrm{P} \text { et } \mathrm{N} \text { (schistes, etc.). } \\
\text { Mal siructurés (limoneux compacts et humides). }\end{array}$ & & Extr. $\mathrm{HCl} 0,2 \mathrm{~N}$. & 3,3 si $\mathrm{pH}>6,4$ \\
\hline Manganèse & $\begin{array}{l}\text { Acides : } \\
\text { - à mauvais drainage et très lessivés (pauvres); } \\
\text { - humifères chaulés }(\mathrm{pH}>6,0) \text { (blocage). } \\
\text { Calcaires : } \\
\text { - craies riches en calcaire actif (crétacé supérieur); } \\
\text { - certains sols argilo-calcaires ; } \\
\text { - tourbes alcalines. }\end{array}$ & $\begin{array}{l}\text { Sols très acidifiés. } \\
\text { Sols acides organi- } \\
\text { ques mal drainés. }\end{array}$ & $\begin{array}{l}\text { Echangeable : } \\
\text { acétate amm.) } \\
\text { pH } 7 . \\
\text { Actif : } \\
\text { échange + facile- } \\
\text { ment réductible } \\
\text { hydroquinone } 0,2 \\
\text { p. } 100 \text {. }\end{array}$ & $\begin{array}{l}-1 \text { si } \mathrm{pH}>6 \\
-20 \text { si pH }>6 \\
\quad \text { (toxique à } 100 \\
\text { pour pH }<5) .\end{array}$ \\
\hline
\end{tabular}

* Cette liste n'est pas limitative, ni obligatoire (tous les sols cités ne sont pas forcément carencés ou toxiques).

** Méthode des Stations I.N.R.A. 
TABLEAU 63. - Oligoéléments et principaux types de sols (pédologie)

\begin{tabular}{|c|c|c|c|c|c|c|}
\hline \multirow{2}{*}{ Groupe de sols } & \multirow{2}{*}{$\begin{array}{l}\text { Conditions } \\
\text { de formation } \\
\text { et caractéristiques }\end{array}$} & \multirow{2}{*}{ Horizons successifs } & \multirow{2}{*}{$\begin{array}{c}\text { Forme des } \\
\text { oligoéléments dans } \\
\text { chaque horizon }\end{array}$} & \multicolumn{2}{|c|}{$\begin{array}{l}\text { Importance des oligoéléments } \\
\text { dans l'horizon }\end{array}$} & \multirow{2}{*}{$\begin{array}{c}\text { Importance } \\
\text { des oligoéléments } \\
\text { disponibles } \\
\text { dans le profil }\end{array}$} \\
\hline & & & & Totaux* & $\begin{array}{c}\text { Mobiles } \\
\text { disponibles* }\end{array}$ & \\
\hline Podzol & $\begin{array}{l}\text { Roche pauvre en Ca, } \\
\text { perméable sous cli- } \\
\text { mat tempéré hu- } \\
\text { mide et végétation } \\
\text { acidifiante (altéra- } \\
\text { tion des argiles } \\
\text { elles-mêmes). }\end{array}$ & $\begin{array}{l}\mathrm{A}_{0} \text { humus brut } \\
\text { (minéral. lente). } \\
\mathrm{A}_{2}: \text { silice pure. } \\
\mathrm{B}_{1}: \text { accum. humus. } \\
\mathrm{B}_{2}: \text { accum. Fe }+++. \\
\mathrm{B}_{3}: \text { accum. argile. } \\
\mathrm{C} \text { : roche acide. }\end{array}$ & $\begin{array}{l}\text { Inclus dans M.O.** } \\
\text { brute (peu minéra- } \\
\text { lisable). } \\
\text { Néant. } \\
\text { Inclus dans M.O. } \\
\text { Inclus dans } \mathrm{Fe}_{2} \mathrm{O}_{3} \text {. } \\
\text { Adsorbés. } \\
\text { Inclus dans miné- } \\
\text { raux. }\end{array}$ & $\begin{array}{l}\text { Assez important. } \\
\text { Néant. } \\
\text { Assez important. } \\
\text { Important. } \\
\text { Assez important. } \\
\text { Plutôt faible. }\end{array}$ & $\begin{array}{l}\text { Très faible. } \\
\text { Nulle. } \\
\text { Nulle. } \\
\text { Nulle. } \\
\text { Moyen. } \\
\text { Faible. }\end{array}$ & $\begin{array}{l}\text { Fonction de la pro- } \\
\text { fondeur de l'hori- } \\
\text { zon } B_{3} \text { (accessible } \\
\text { ou non aux raci- } \\
\text { nes). }\end{array}$ \\
\hline Sol lessivé & $\begin{array}{l}\text { Roche pauvre en Ca, } \\
\text { moyennement fil- } \\
\text { trante sous climat } \\
\text { et végétation peu } \\
\text { acidifiante (pas } \\
\text { d'altération des ar- } \\
\text { giles). }\end{array}$ & $\begin{array}{l}\mathrm{A}_{0} \text { : humus doux } \\
\text { (minéral. rapide). } \\
\mathrm{A}_{1} \text { : appauvri en } \\
\text { argile. } \\
\mathrm{B}: \text { accum. argile et } \\
\text { fer. } \\
\mathrm{C}: \text { roche acide. }\end{array}$ & $\begin{array}{l}\text { Inclus dans M.O. } \\
\text { (très minéralis.). } \\
\text { Adsorbés. } \\
\text { Inclus et adsorbé. } \\
\text { Inclus et adsorbé } \\
\text { sur argile et fer. } \\
\text { Inclus dans miné- } \\
\text { raux. }\end{array}$ & $\begin{array}{l}\text { Important. } \\
\text { Faible. } \\
\text { Important. } \\
\text { Plutôt faible. }\end{array}$ & $\begin{array}{l}\text { Moyen. } \\
\text { Plutôt faible. } \\
\text { Assez riche. }\end{array}$ & $\begin{array}{l}\text { Fonction de la pro- } \\
\text { fondeur de } \mathrm{B} \text {, mais } \\
\text { moins appauvri } \\
\text { que dans les pod- } \\
\text { zols. }\end{array}$ \\
\hline Sol brun & $\begin{array}{l}\text { Roches dures non } \\
\text { calcaires sous cli- } \\
\text { mat tempéré peıt } \\
\text { humide, végétation } \\
\text { peu acidifiante } \\
\text { (oxydes de fer liés } \\
\text { au complexe argilo- } \\
\text { humique floculé). }\end{array}$ & $\begin{array}{l}\mathrm{A}_{1}: \text { humus doux. } \\
\mathrm{B} \text { : homogène. } \\
\mathrm{BC}: \text { riche en oxyde } \\
\text { de fer. } \\
\mathrm{C}: \text { roche non } \\
\text { calcaire. }\end{array}$ & $\begin{array}{l}\text { Inclus dans M.O. } \\
\text { (très minéralis.). } \\
\text { Adsorbés. } \\
\text { Partiellement inclus } \\
\text { dans hydr. } \\
\text { Adsorbés. }\end{array}$ & Assez homogène. & Assez riche. & Riche. \\
\hline
\end{tabular}


TABLEAU 63 (suite.) - Oligoéléments et principaux types de sols (pédologie)

\begin{tabular}{|c|c|c|c|c|c|c|}
\hline \multirow{2}{*}{ Groupe de sols } & \multirow{2}{*}{$\begin{array}{l}\text { Conditions } \\
\text { de formation } \\
\text { et caractéristiques }\end{array}$} & \multirow{2}{*}{ Horizons successifs } & \multirow{2}{*}{$\begin{array}{l}\text { Forme des } \\
\text { oligoéléments dans } \\
\text { chaque horizon }\end{array}$} & \multicolumn{2}{|c|}{$\begin{array}{c}\text { Importance des oligoéléments } \\
\text { dans l'horizon }\end{array}$} & \multirow{2}{*}{$\begin{array}{c}\text { Importance } \\
\text { des oligoéléments } \\
\text { disponibles } \\
\text { dans le profil }\end{array}$} \\
\hline & & & & Totaux* & $\begin{array}{l}\text { Mobiles } \\
\text { disponibles* }\end{array}$ & \\
\hline $\begin{array}{c}\text { Sol } \\
\text { hydromorphe } \\
\text { à } \\
\text { pseudogley }\end{array}$ & $\begin{array}{l}\text { Couche imperméa- } \\
\text { ble, d'où ennoyage } \\
\text { temporaire (mini- } \\
\text { mum } 6 \text { mois sur } \\
\text { 12). }\end{array}$ & $\begin{array}{l}\mathrm{A}_{1}: \text { humus spécial. } \\
\mathrm{A}_{1} \mathrm{~g}: \text { stagnation } \\
\text { temporaine de l'eau } \\
\text { (taches grises et } \\
\text { rouilles). } \\
\text { (B)g : enrichi ou } \\
\text { non en argile, mar- } \\
\text { morisé. } \\
\mathrm{C}: \text { indifférent. }\end{array}$ & $\begin{array}{l}\text { Inclus dans concré- } \\
\text { tions ferriques - } \\
\text { (solubilisation par- } \\
\text { tielle sous ennoya- } \\
\text { ge). }\end{array}$ & $\begin{array}{l}\text { Appauvri par } \\
\text { rapport à C. }\end{array}$ & $\begin{array}{l}\text { Répartition hétéro- } \\
\text { gène non entre ho- } \\
\text { rizons mais entre } \\
\text { parties blanches et } \\
\text { rouilles, variable } \\
\text { avec l'époque fonc- } \\
\text { tion de l'ennoyage. }\end{array}$ & $\begin{array}{l}\text { Très appauvri (éli- } \\
\text { mination en pério- } \\
\text { de d'ennoyage). }\end{array}$ \\
\hline $\begin{array}{c}\text { Sols } \\
\text { à } \\
\text { sesquioxydes }\end{array}$ & $\begin{array}{l}\text { Climat tropical ( } \pm \\
\text { atténué) à saisons } \\
\text { alternativement hu- } \\
\text { mide et sèche, for- } \\
\text { te évaporation pro- } \\
\text { voquant des mouve- } \\
\text { ments ascendants, } \\
\text { végétation peu dé- } \\
\text { veloppée. }\end{array}$ & $\begin{array}{l}\text { A | oxydes ferriq. } \\
\text { (B) | deshydratés. } \\
\text { C : divers. }\end{array}$ & $\begin{array}{l}\text { Inclus dans les oxy- } \\
\text { des. } \\
\text { Adsorbés. }\end{array}$ & Riche. & $\begin{array}{l}\text { Très faible (dépend } \\
\text { de l'argile). }\end{array}$ & Très pauvre. \\
\hline $\begin{array}{l}\text { Rendzines } \\
\text { grisels }\end{array}$ & $\begin{array}{l}\text { Roches calcaires } \\
\text { tendres (mélange } \\
\text { intime entre les élé- } \\
\text { ments minéraux et } \\
\text { organiques), climat } \\
\text { indifférent, plutôt } \\
\text { sec. }\end{array}$ & $\begin{array}{l}\text { A : calcaire, argile } \\
\text { et humus (forte } \\
\text { activité). } \\
\text { C : calcaire tendre. }\end{array}$ & $\begin{array}{l}\text { Inclus dans le com- } \\
\text { plexe argile-humate } \\
\text { oalcique, énergique- } \\
\text { ment floculé. }\end{array}$ & $\begin{array}{l}\text { Abondant. } \\
\text { Variable. }\end{array}$ & $\begin{array}{l}\text { Souvent faible. } \\
\text { Variable. }\end{array}$ & Souvent faible. \\
\hline
\end{tabular}

* Appréciation: de la roche-mère (horizon C) par rapport à Ja moyenne des roches.

** M.O. = Matière organique.

des horizons A et B par rapport à la roche-mère du profil. 
On doit à Lamand et Périgaud (I.N.R.A.-C.R.Z.Y. Theix) une étude sur la pauvreté des sols français en certains oligoéléments. Ils ont pu établir une carte des sols pauvres en cuivre et en cobalt ; " mais elle présente de nombreuses lacunes par suite d'une absence totale de données tant au niveau des roches, surtout sédimentaires, que des sols".

Des carences en cuivre et zinc sont à attendre dans presque tous les départements français. Des carences en manganèse sont à attendre sur les sédiments calcaires déjà carencés en cuivre et cobalt :

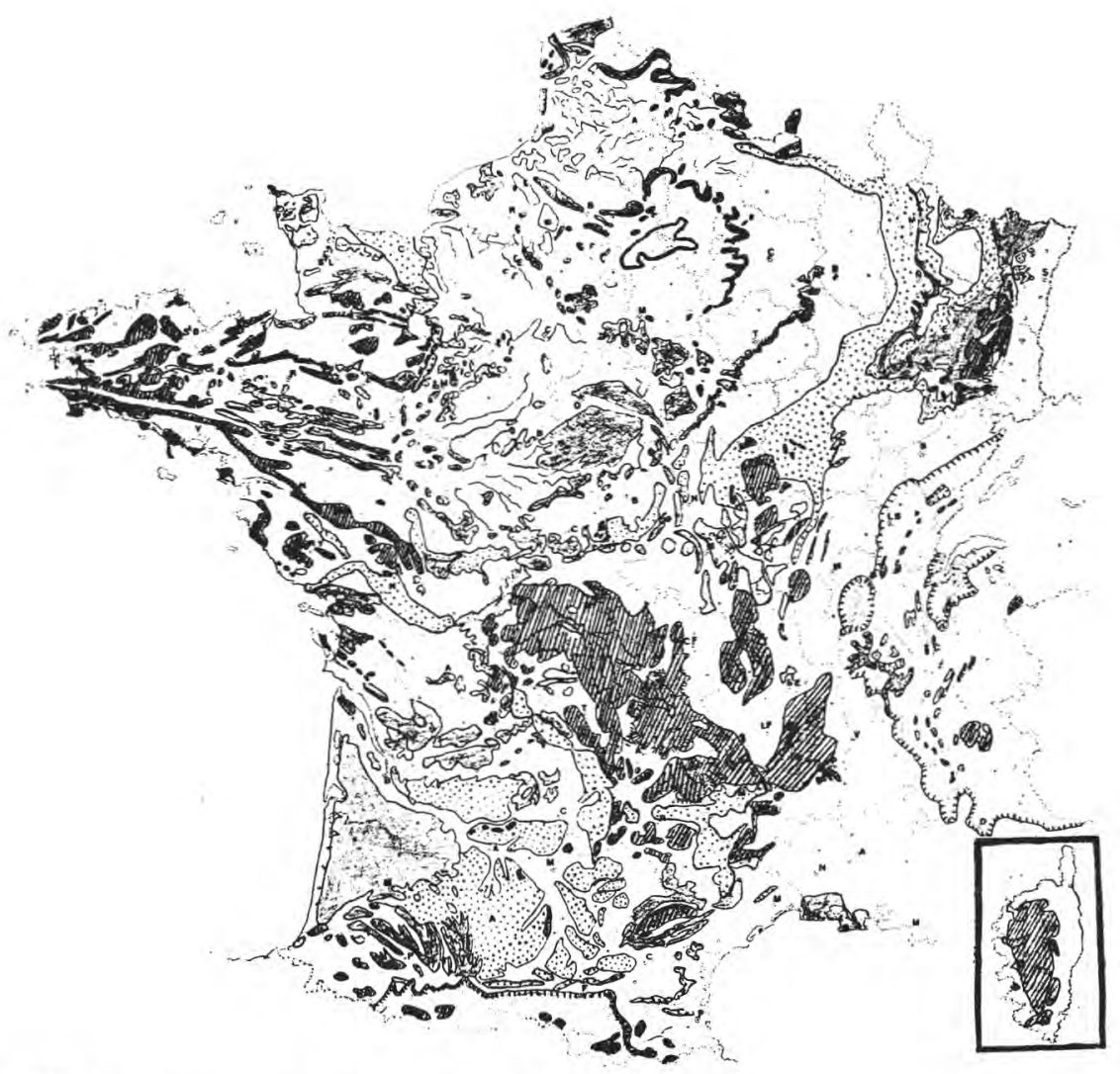

Roches éruptives et métamorphiques

Roches sédimentaires siliceuses

ars + argiles riches en molybdene

$\because \because$ Calcaires et molasses

fig. 5

Carte des sols pauvres en cuivre et cobalt en France 
- dans les terres calcaires riches en matière organique et ferrugineuse (du type rendzines) ou les sols rouges méditerranéens plus ou moins décalcarifiés,

- dans les terres humifères acides dont on relève trop le $\mathrm{pH}$ par chaulage.

I1 y a aggravation des carences par intensification fourragère, lorsque la fertilisation est importante.

\subsection{Oligoéléments et fourrages}

De nombreuses études ont été effectuées sur la composition des fourrages en certains oligoéléments, en fonction du stade de végétation, de la nature du sol, de la fertilisation. Ne sont mentionnés ici que deux tableaux :

- le tableau 64 résume les travaux de Lamand et Périgaud sur les végétaux fourragers en spécifiant les aires de carence et comment y remédier,

- le tableau 65 définit les seuils de carence et de toxicité comparés pour la plante et le ruminant.

Les foins sont pauvres en cuivre si on adopte le seuil de $5 \mathrm{mg} / \mathrm{kg}$; ils sont tous pauvres en zinc.

Des teneurs en manganèse inférieures à $40 \mathrm{mg} / \mathrm{kg}$ de matière sèche ne se rencontrent que dans des zones très limitées.

\subsection{Oligoéléments et ruminants}

Les facteurs conditionnant les besoins des animaux en oligoéléments et la couverture de ces besoins sont rassemblés dans la figure 6.

Des seuils de carence et de toxicité ont été établis pour différents éléments : pour le cuivre, le manganèse et le zinc, tableau 65 .

\subsubsection{Le cuivre}

La carence des sols en cuivre (tab. 64) existerait en Bretagne, en Basse-Normandie et dans le Bas-Maine.

En cas de carence en cuivre, l'appétit diminue à l'égard de l'herbe et du foin, mais demeure inchangé vis-à-vis des concentrés ; le poil se décolore, une diarrhée toujours violente, de couleur jaune-verdâtre et d'odeur nauséabonde attire l'attention. On observe chez la vache une baisse de la production laitière due à la dénutrition, elle-même provoquée par la perte d'appétit. La fécondité des vaches diminue.

L'influence de la carence en cuivre sur la fécondité a été observée chez des vaches où cette carence était conditionnée pour une teneur excessive des fourrages en molybdène : le molybdène perturbe l'absorption du phosphore dont on connaît l'action favorable sur la reproduction, chez les vaches en particulier. 
TABLEAU 64. - Oligoéléments et végétaux (spécialement fourrages) [d'après Lamand et Périgaud]

\begin{tabular}{|c|c|c|c|c|c|}
\hline & \multirow{2}{*}{$\begin{array}{l}\text { Plantes sensibles } \\
\text { à la carence }\end{array}$} & \multirow{2}{*}{$\begin{array}{l}\text { Aires de carence } \\
\text { connues en France* }\end{array}$} & \multicolumn{2}{|c|}{ Teneur des foins, $\mathrm{mg} / \mathrm{kg}$} & \multirow{2}{*}{$\begin{array}{c}\text { Méthodes possibles } \\
\text { d'enrichissement des } \\
\text { fourrages }\end{array}$} \\
\hline & & & $\begin{array}{c}\text { Moyenne des minimas } \\
\text { et maximas }\end{array}$ & $\begin{array}{l}\text { Minimum admissible } \\
\text { pour les animaux }\end{array}$ & \\
\hline Cuivre & $\begin{array}{l}\text { Céréales (sauf seigle) : } \\
\text { Ray-grass, fléole, trè- } \\
\text { fle blanc sélectionné, } \\
\text { trèfle violet. }\end{array}$ & $\begin{array}{c}\text { Bretagne, Basse-Nor- } \\
\text { mandie, Bas-Maine. }\end{array}$ & $1-20$ & $\begin{array}{c}5 \text { à } 10 \\
\text { sselon le taux de Mo }\end{array}$ & $\begin{array}{l}\text { Epandage } \mathrm{CuSO}_{4} 5 \mathrm{H} 20 \text { : } \\
2,5 \mathrm{~kg} \text { toutes les } 3 \\
\text { semaines (Pays-Bas) } \\
30-50 \mathrm{~kg} / \text { an. } \\
\text { Pulvérisations? }\end{array}$ \\
\hline Manganèse & $\begin{array}{l}\text { Céréales. } \\
\text { Betteraves. } \\
\text { Fruitiers. }\end{array}$ & $\begin{array}{l}\text { Bretagne, Nord, Cham- } \\
\text { pagne, Roussillon, } \\
\text { Vallée de la Loire. }\end{array}$ & $10-1000$ & $\begin{array}{l}25 \text { à } 150 \\
\text { selon auteurs }\end{array}$ & $\begin{array}{l}\text { Sulfate manganèse : } 5 \\
\mathrm{~kg} / \mathrm{ha} \text { tcus les mois ? } \\
\text { Pulvérisations ? }\end{array}$ \\
\hline Zinc & Maïs, lin, fruits. & $\begin{array}{l}\text { S.O. Roussillon, Breta- } \\
\text { gne, Vallée de ia Loi- } \\
\text { re, Haute-Normandie. }\end{array}$ & $10-100$ & 20 à 40 & Non connu \\
\hline
\end{tabular}

* Carences effectivement démontrées. 
TABLEAU 65. - Les oligoéléments dans les fourrages. Seuils de carence et de toxicité comparés pour la plante et le ruminant

\begin{tabular}{|c|c|c|c|c|c|c|}
\hline & \multicolumn{3}{|c|}{ Fourrages } & \multicolumn{3}{|c|}{ Ruminants } \\
\hline & $\begin{array}{l}\text { Seuil de carence } \\
\text { p.p.m. MS }\end{array}$ & $\begin{array}{l}\text { Seuil de toxicité } \\
\text { p.p.m. MS* }\end{array}$ & $\begin{array}{l}\text { Teneurs normales } \\
\text { et (extrêmes) }\end{array}$ & $\begin{array}{l}\text { Seuil de toxicité } \\
\text { p.p.m. MS } \\
\text { de la ration }\end{array}$ & $\begin{array}{c}\text { Seuil de carence } \\
\text { p.p.m. MS } \\
\text { de la ration }\end{array}$ & $\begin{array}{c}\text { Interférences } \\
\text { connues }\end{array}$ \\
\hline $\mathrm{Cu}$ & $2-4$ & $>20$ & $4-15$ & $\begin{array}{c}\text { Bovin adulte } \\
410 \\
\text { Jeune bovin } \\
+ \text { sensible } \\
\text { Ovin } \\
\text { rustique } \\
4-6 \\
\text { Mérinos } \\
7-8\end{array}$ & $\begin{array}{c}\text { Ovin } \\
20-30 \\
\text { Jeune bovin } \\
40-100 \\
\text { Bovin adulte } \\
<200\end{array}$ & $\begin{array}{l}\mathrm{Cu}-\mathrm{Mo} \\
\mathrm{Cu}-\mathrm{SO}_{4} \\
\mathrm{Cu}-\mathrm{Ca} \\
\mathrm{Cu} ? \\
\mathrm{Cu}-\mathrm{Zn} \\
\mathrm{Cu}-\mathrm{N}\end{array}$ \\
\hline Mn & & $\begin{array}{c}\text { Fonction } \\
\mathrm{du} \text { rapport } \mathrm{Fe} / \mathrm{Mn}\end{array}$ & $\begin{array}{c}15-200 \\
\text { (jusqu’à } 600 \text { ) }\end{array}$ & $20-50$ & Inconnu & $\mathrm{Mn}-\mathrm{Ca}$ \\
\hline $\mathrm{Zn}$ & $8-15$ & $>200$ & $8-50$ & $\begin{array}{c}\text { Adulte } \\
40-45 \\
\text { Jeune } \\
+ \text { sensible }\end{array}$ & $500-900$ & $\begin{array}{c}\text { Zn-phytates } \\
\text { Zn-Ca } \\
\mathrm{Zn}-\mathrm{Cu} \\
\mathrm{Zn}-\mathrm{Mn}\end{array}$ \\
\hline
\end{tabular}

Références tirées de Underwood et Allaway.

* Les toxicités $\mathrm{Cu}-\mathrm{Mn}-\mathrm{Zn}$ sont connues sur végétaux, mais correspondent plus à un ralentissement de croissance et des symptômes spécifiques qu'à des teneurs définies dans la plante (Stiles). 


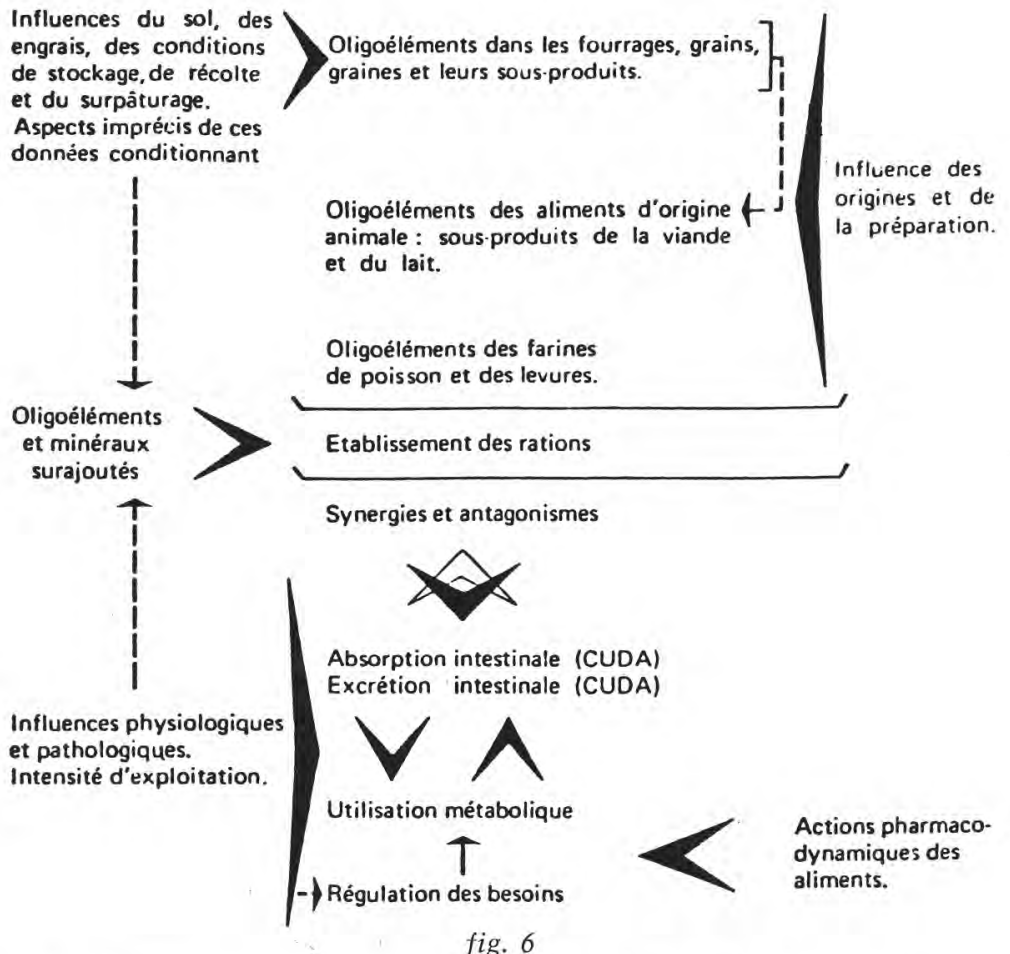

Facteurs conditionnant les besoins des animaux en oligoéléments et la couverture de ces besoins

Des troubles cardiaques surviennent progressivement chez les sujets carencés, pouvant entraîner une mort subite. Il existe souvent une anémie, le cuivre étant utile pour libérer le fer stocké dans le foie.

Le cuivre intervient dans les phénomènes d'oxydations biologiques essentiels au métabolisme des tissus de soutien et du collagène. Le cuivre n'existe pas à l'état ionisé libre dans les différents tissus à l'exception du tube digestif : il y jouerait un rôle analogue à celui des antibiotiques, dans l'alimentation. Partout ailleurs le cuivre est lié à des protéines: les cuproprotéines (céruloplasmine, cuproprotéines tissulaires, cuproenzymes). Il jouerait un rôle important dans la manifestation du sens gustatif.

Le cuivre a biologiquement des relations avec le fer, le cobalt, le zinc, le manganèse, le phosphore et le soufre. Une certaine quantité de fer est nécessaire pour que le cuivre remplisse son rôle ; il faut un apport de cuivre pour libérer le fer stocké ; le fer en excès pourrait perturber l'absorption de cuivre. Le zinc agit de même, mais 
sa propre absorption est défavorablement influencée par un excès de cuivre.

Les sulfates diminuent l'absorption du cuivre, mais également celle du molybdène. Il y aurait chez les ruminants formation de sulfure de cuivre insoluble dans le rumen.

Le molybdène forme avec le cuivre des complexes inhibiteurs de son activité biochimique. Il rendrait le cuivre non utilisable par l'organisme au niveau des tissus. Inversement, il semble qu'une carence en molybdène rendrait plus aisée l'intoxication par le cuivre.

Le cuivre serait toxique à la dose de $250 \mathrm{mg}$ par jour. Des intoxications ont été observées lors de la consommation d'herbes provenant de pâturages traités au sulfate de cuivre afin de détruire certains hôtes intermédiaires de parasites, ou de prairies ayant reçu de l'oxychlorure de cuivre.

\subsubsection{Le manganèse}

La carence des sols en manganèse existerait en Bretagne, dans le Nord de la France, en Champagne, dans le Roussillon et dans la Vallée de la Loire (tab. 64).

Cette carence retentit sur le squelette en entraînant des déformations osseuses, y compris l'apparition d'un jaret rectiligne. Elle provoque une altération de la fonction sexuelle et des phénomènes d'hyperexcitabilité et d'atoxie dus à des malformations osseuses du labyrinthe.

Les besoins de la vache laitière sont estimés par excès à $22 \mathrm{ppm}$. Le manganèse jouerait un rôle d'activateur des enzymes phosphorylantes notamment au niveau de l'A.T.P., il serait un activateur de l'arginase des peroxydases, des phosphatases et des peptidases. Il a une action dans le développement osseux.

Le rapport $\mathrm{Mn} / \mathrm{Ca}$ du poil a une corrélation positive avec la teneur des herbages en manganèse ; il est constant pendant la saison de pâturage.

Il y a interférence entre l'absorption du manganèse, celle du fer, celle du calcium et des phosphates de chaux.

\subsubsection{Le zinc}

On a signalé un manque de zinc dans les terres du Sud-Ouest, du Roussillon, de la Vallée de la Loire et de la Haute-Normandie. Les accidents de carence sont constatés sur du bétail consommant du maïs. Le maïs souffre par suite d'une limitation de l'azote absorbé par la plante (tab. 64).

Les fourrages verts contiennent entre 30 et $100 \mathrm{ppm}$ de zinc par $\mathrm{kg}$ de matière sèche. Un bétail consommant du fourrage en contenant 20 à $80 \mathrm{mg} / \mathrm{kg}$ de matière sèche a montré des signes de carence : lésions cutanées, alopécie périoculaire et sur le cou ; 
perte de fécondité et baisse de la lactation. Corrigé par un apport de zinc dans la ration.

La zincémie reste sous la dépendance du taux de calcium de la ration. Elle s'abaisse quand le calcium des aliments s'élève. La teneur en zinc de poils reflète celle du régime.

Le zinc fait partie de la composition de nombreuses métalloenzymes. Il permettrait l'enroulement et le déroulement de la double hélice d'A.D.N., sous l'influence de faibles changements de température.

L'excès de calcium serait un facteur d'importance pour favoriser l'apparition de la carence en zinc, comme son aggravation ; le cadmium est un antagoniste puissant du zinc ; le lactose favoriserait l'absorption du zinc. La vitamine D qui agit sur l'absorption du calcium, élève également celle du zinc, entraînant une augmentation de cet oligoélément dans les os.

Les besoins en zinc augmenteraient avec le taux des protéines de la ration. Des taux élevés de zinc gènent l'absorption du cuivre et du fer ; il accélérerait l'élimination de ce dernier métal. Si cela se confirmait, cela constituerait un moyen de produire des veaux blancs.

La mise en évidence en Bretagne et dans le Sud-Ouest de carence du maïs en zinc oblige à admettre l'éventualité de l'apparition d'accidents sur le bétail français qui consomme de plus en plus cette céréale en ensilage, et en grains. D'une façon générale, l'élévation du $\mathrm{pH}$ des sols due à des apports souvent trop copieux d'amendements calcaires, entraîne une moindre teneur des plantes en zinc.

Il importe d'ajouter un supplément de zinc aux rations des animaux ; ce qui permet dans une faible mesure d'augmenter le taux de zinc dans le lait. Mais la glande mammaire ne capte qu'une faible partie du zinc sérique ; elle exerce une sorte de discrimination. Quant à la viande, sa teneur ne varie guère.

\subsubsection{Relations entre les éléments de la ration}

L'étude des trois éléments précédents a permis de voir la complexité des liaisons qui existent entre les minéraux ; mais d'autres liaisons sont établies entre les minéraux et les enzymes, les vitamines ou les antibiotiques.

La figure 7 est une représentation des relations qui peuvent exister entre les éléments minéraux de la ration.

\subsubsection{Carences en oligoéléments chez les ruminants}

Le tableau 66 rassemble pour sept oligoéléments les principaux symptômes de carence que l'on peut observer, pouvant amener à un diagnostic de carence.

Il faut noter que ne peuvent être pris en considération pour le diagnostic, les déficits de productions : croissance, engraissement, 
Relations entre les éléments minéraux de la ration

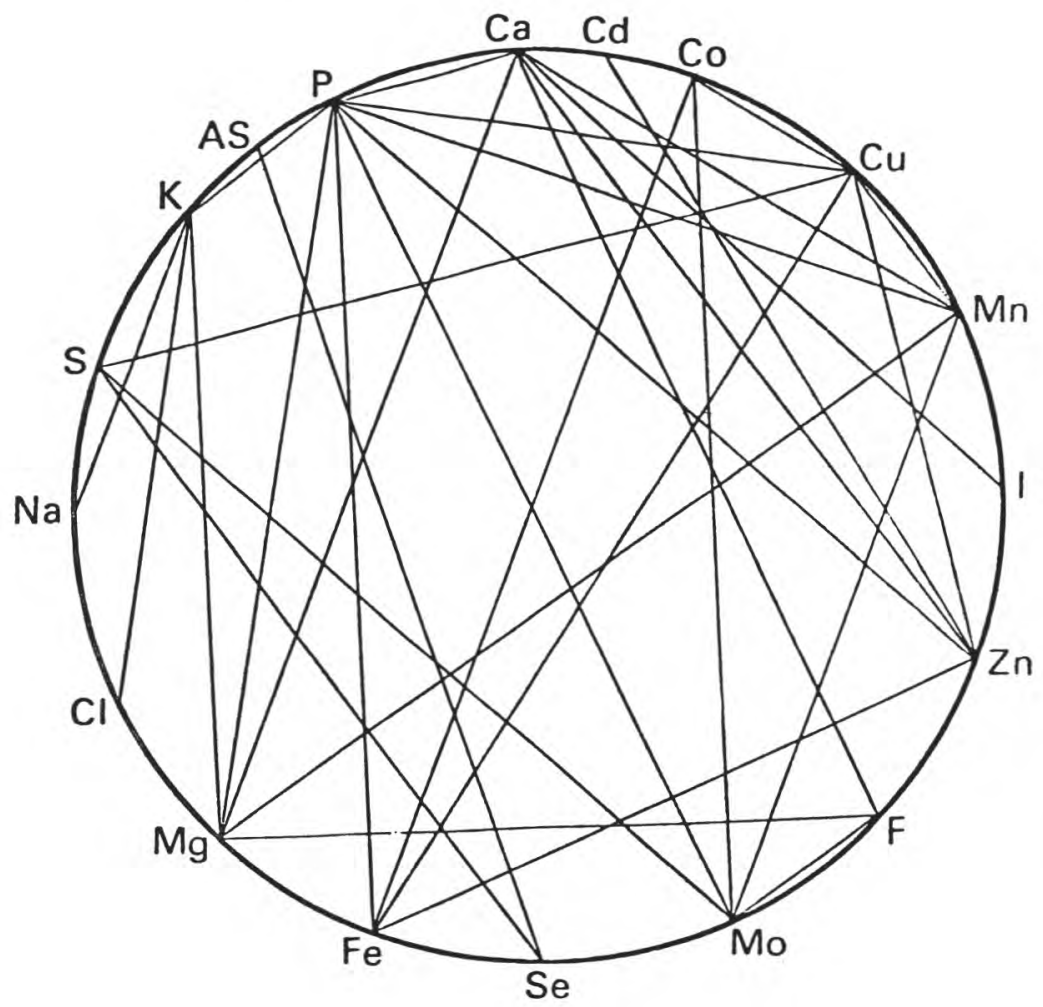

fig. 7

D'après « Mineral Interrelations » (G. B. Ammerman, 1965) avec quelques modifications dues à Ferrando

lactation, reproduction. Les symptômes n'apparaissent jamais ensemble sur un même animal et varient d'un malade à l'autre.

On se rend compte finalement que, du fait de ces interactions connues, ou inconnues, pratiquement, on ne peut rien prévoir pour remédier à priori à une carence. On constate l'hétérogénéité du sol, que la fertilisation et les techniques culturales modifient la teneur et le pourcentage des oligoéléments assimilables d'un sol par les plantes.

Cependant, en reliant l'ensemble de ces études avec les résultats des analyses de fourrages, il est possible d'améliorer l'équilibre des rations. 
TABLEAU 66. - Symptômes des canences en oligoéléments chez les ruminants

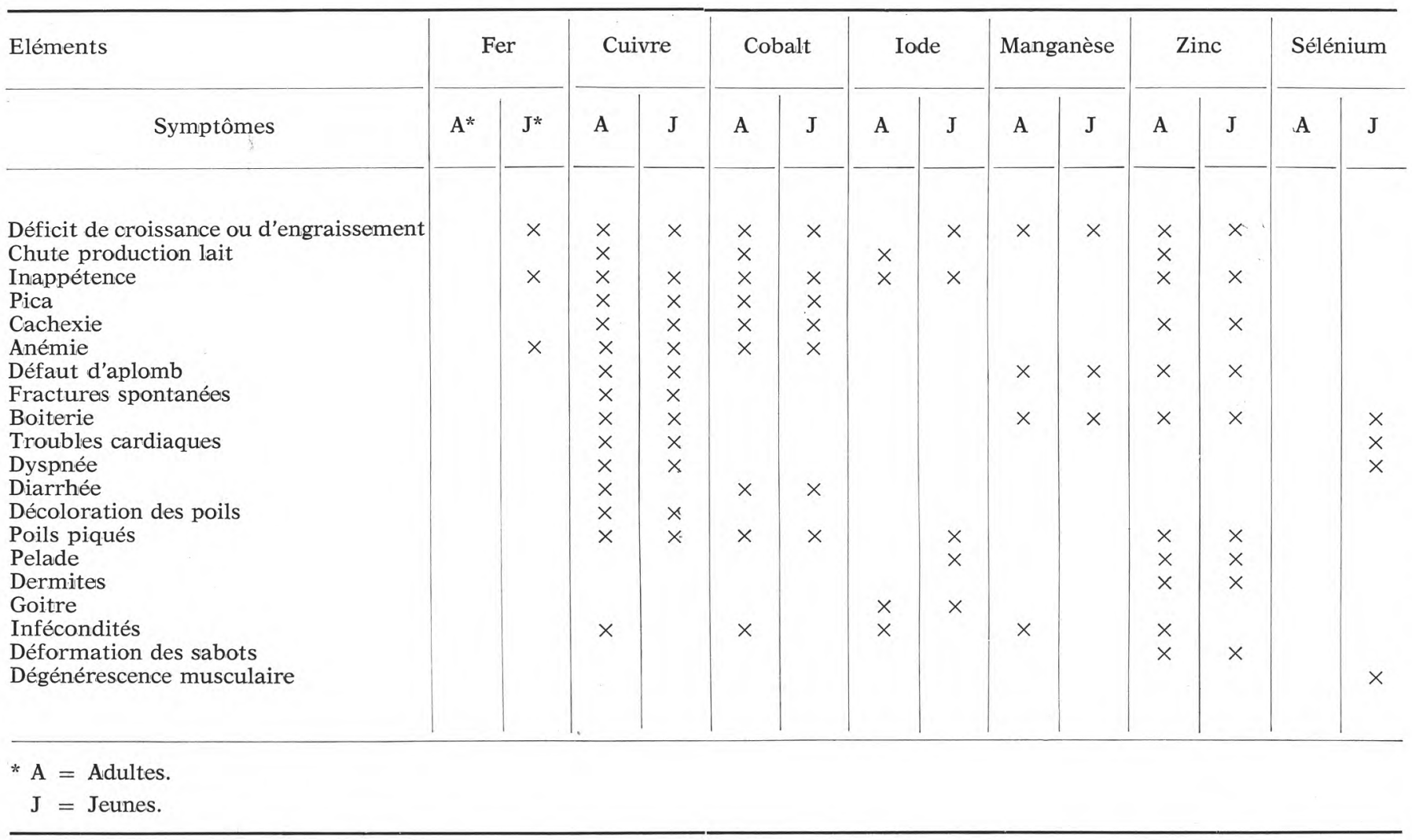




\subsection{Oligoéléments et l'homme}

\subsubsection{Le fer}

L'organisme humain n'utilise que 5 à 10 p. $100 \mathrm{du}$ fer que lui apportent les aliments. Ainsi, bien que les besoins soient estimés à $1-2,5 \mathrm{mg} / \mathrm{j}$, les doses recommandées selon l'âge et l'état physiologique par jour sont de 6 à $15 \mathrm{mg}$.

L'ingestion dépasse facilement les doses minimales. L'excès n'entraîne aucun inconvénient car le fer, non immédiatement utilisé par l'organisme est mis en réserve sous la forme de ferroprotéines (ferritine et hémosidérine) principalement dans le foie, la rate et la moelle épinière. Les accumulations de fer qui ont pu être décrites sont le fait de troubles du métabolisme plutôt que de l'ingestion de quantités excessives de fer.

\subsubsection{Le cuivre}

Les besoins quotidiens sont pour l'homme de l'ordre de $2 \mathrm{mg}$; selon le régime, des quantités notablement plus élevées peuvent être ingérées, ce qui a pour effet d'accroître l'excrétion surtout fécale. Une partie du cuivre est cependant retenue dans le foie. L'absorption et la rétention dépendent aussi bien de la forme sous laquelle le cuivre est présent dans les aliments que des doses de molybdène, d'ion sulfate et de zinc qu'apporte le régime.

Le Comité mixte F.A.O./O.M.S. d'Experts des Additifs alimentaires (réf. 8, p. 16) a indiqué comme admissible un apport maximal quotidien de $0,5 \mathrm{mg}$ de cuivre par $\mathrm{kg}$ de poids corporel $(30 \mathrm{mg} / \mathrm{j}$ pour un homme de $60 \mathrm{~kg}$ ) sous réserve que le régime soit par ailleurs normal, notamment en ce qui concerne sa teneur en molybdène et en zinc.

On a signalé des intoxications aiguës par des doses élevées (plusieurs grammes) de sels de cuivre, ainsi que des intoxications professionnelles d'ouvriers exposés à des poussières ou à des vapeurs cuivriques. Il n'a jamais été observé un effet à longue échéance du cuivre ingéré en faibles doses avec les aliments (même au temps $\mathrm{du}$ reverdissage des légumes en conserve, jusqu'à $100 \mathrm{mg}$ de $\mathrm{Cu} / \mathrm{kg}$ d'aliment) ni d'action cancérogène.

Le cuivre, en tant qu'oligoélément, est dénué d'action toxique.

\subsubsection{Le zinc}

La dose journalière indispensable à l'homme est de 0,2 à $0,3 \mathrm{mg} / \mathrm{kg}$ de poids corporel ; mais certains régimes en fournissent des doses bien plus élevées sans entraîner le moindre inconvénient. Les teneurs en cuivre, fer et molybdène interviennent également de telle sorte qu'il est impossible d'indiquer des chiffres précis sans tenir compte des autres éléments présents.

Des intoxications aiguës ont été observées provoquées soit par l'absorption, accidentelle ou criminelle de doses massives (plusieurs 
grammes) de sels de zinc, soit par l'ingestion d'aliments acides qui avaient été cuits ou gardés dans des récipients en fer galvanisé (600 à $800 \mathrm{mg} / \mathrm{kg}$ d'aliment). On ne connaît pas d'exemple de toxicité à long terme.

Le zinc, en tant qu'oligoélément, est dénué de toxicité.

\subsubsection{Le manganèse}

On n'a jamais signalé de carence en manganèse chez l'homme. Les doses journalières nécessaires sont de l'ordre de 0,1 mg par $\mathrm{kg}$ de poids corporel. Les régimes habituels, s'ils sont suffisamment variés, en apportent le nécessaire.

On a observé des intoxications par les poussières de bioxyde de manganèse chez les ouvriers travaillant à l'extraction du minerai. Mais en dehors d'accidents aigus dus à l'absorption de doses massives de sels de manganèse notamment permanganate de potassium, jamais il n'a été signalé d'effets à long terme de petites doses de manganèse per os.

Malgré les lacunes dans les données expérimentales, il semble certain que le manganèse, en tant qu'oligoélément, est dénué de toxicité.

\subsubsection{Le magnésium}

Les doses nécessaires à l'organisme humain sont de 250 à 400 $\mathrm{mg} / \mathrm{j}$, c'est-à-dire plus proches des doses de calcium (de l'ordre du gramme) que de celles des vrais oligoéléments.

Le magnésium ne pose pas de problème toxicologique ; cependant des doses excessives dans le régime peuvent entraîner, comme avec le calcium, des troubles graves au niveau des reins.

\subsubsection{Le plomb}

L'homme préhistorique ingérait environ $0,02 \mathrm{mg} \mathrm{Pb} / \mathrm{j}$, essentiellement avec les aliments; l'homme d'aujourd'hui en ingère 0,41 à $0,46 \mathrm{mg} / \mathrm{j}$, dont la moitié provient des aliments et l'autre moitié de l'air des villes et de la fumée de tabac. La plombémie actuelle du citoyen américain est dangereusement proche des 0,5 à $0,8 \mathrm{mg} / \mathrm{l}$, où se situe le seuil du saturnisme.

Environ 90 p. 100 du plomb introduit par voie digestive se retrouve dans les fécès; l'absorption varie considérablement selon la forme sous laquelle le plomb est ingéré. Seulement 5 p. 100 du plomb apporté par les aliments est absorbé; les 0,3 à $0,4 \mathrm{mg}$ que nous ingérons quotidiennement, sont donc sans danger. Il faut cependant s'efforcer de réduire cet apport autant que possible à cause de l'importance de la pollution atmosphérique, sous ses diverses formes.

\subsubsection{L'arsenic}

La France est parmi les pays les plus riches en minerais d'arsenic : on en trouve principalement dans l'Aude (Montagne Noire), en 
Corse (gisement de Matra), dans la Creuse, la Haute-Vienne, le Maine-et-Loire, la Mayenne, les Pyrénées-Orientales et les Vosges. La terre arable en renferme normalement quelques milligrammes par $\mathrm{kg}$.

L'arsenic se rencontre dans les eaux $(0,01$ à $0,1 \mathrm{mg} / \mathrm{l}$ dans l'eau de mer), dans l'atmosphère : des traces faibles sauf dans le cas de pollutions locales industrielles (Salsigné). Les aliments consommés par l'homme contiennent donc toujours de l'arsenic. La Société des Experts Chimistes de France a cherché à fixer les teneurs qui pouvaient être acceptées compte tenu des contaminations susceptibles de se produire lors de la préparation des denrées alimentaires : $0,05 \mathrm{mg} / 1$ pour le lait ; $0,2 \mathrm{mg} / 1$ pour les boissons...

La toxicité des composés arsénicaux varie beaucoup selon leur nature, les composés organiques oxygénés étant parfois très peu toxiques. Par exemple les toxicités aiguës en injection sous-cutanée de l'anhydride arsénieux ou de l'arséniate de potassium sont respectivement environ 250 et 100 fois plus élevées que celles du cacodylate de sodium ou de méthyl arsinate de sodium. Cette toxicité se manifeste par des troubles gastro-intestinaux, une perte de poids, des éruptions cutanées, des polynévrites, etc.

Si des animaux ont été intoxiqués soit du fait de contaminations par fumées arsénicales, soit à la suite de traitements insecticides, il n'en est pas résulté d'accidents pour l'homme consommant leur viande. Par contre, de nombreux accidents ont résulté de l'utilisation d'une farine souillée par des composés arsénicaux, dans les préparations de pain et de gâteaux.

La fréquence des accidents a maintenant considérablement diminué, grâce au contrôle des critères de pureté de tous ces produits utilisés en industrie alimentaire (l'acide sulfurique notamment) et aux précautions prises lors des traitements insecticides.

\subsubsection{Le mercure}

Les traces sont habituellement très faibles chez les végétaux et les animaux. La quantité de mercure ingérée journellement par l'homme en provenance de son alimentation a été estimée de 0,005 à $0,02 \mathrm{mg}$. Le danger vient des effets cumulatifs par lesquels il manifeste sa toxicité ; cependant aux doses estimées ci-devant, l'accumulation tissulaire est encore très faible. Les experts du Comité mixte F.A.O./O.M.S. des additifs alimentaires estiment que la contamination du milieu ambiant par le mercure est en train de s'aggraver.

Le mercure lui-même est surtout dangereux par inhalation, il entraîne des accidents buccaux, des troubles nerveux et des lésions rénales. Par contre, il peut être ingéré en quantités importantes sans cause de troubles, à moins qu'il n'ait été finement divisé.

La toxicité des sels de mercure paraît liée à leur solubilité :

- le vermillon (sulfure de $\mathrm{Hg}$ ) insoluble est inoffensif ; 
- le calomel (chlorure mercureux) peu soluble, est peu toxique (purgatif) ;

- le sublimé corrosif (chlorure mercurique) est très toxique (puissant antiseptique) ;

- les organomercuriels utilisés en agriculture comme fongicides sont très toxiques : dérivés méthylés, éthylés, phénylés et tolylés.

La concentration de mercure chez les poissons, même chez ceux péchés en mer, dans les lacs ou dans des rivières que l'on croit non pollués reste plus élevée que celle de la plupart des autres denrées alimentaires (le foie de certains poissons transformerait le mercure en méthyl-mercure). La situation est particulièrement préoccupante au Japon où des intoxications très graves s'y sont produites : Minimata et Niigata.

\subsubsection{Le fluor}

Le fluor est un élément particulièrement répandu dans la nature. On le trouve notamment dans le sol et dans l'eau, d'où la fréquence des intoxications hydro-telluriques (fluorose du bétail). Le sol en renferme de 0 à $150 \mathrm{mg}$ p. 100 ; la teneur augmente avec la profondeur ; le taux peut atteindre $700 \mathrm{mg}$ p. 100 lors d'emploi d'engrais phosphatés.

L'air peut être contaminé au voisinage des usines d'aluminium (vapeurs et poussières fluorées) et dans les villes lors de la combustion de charbon ou de schistes dont le taux de fluor peut atteindre 4 à $5 \mathrm{mg}$ p. 100 .

Les causes d'intoxications fluorées sont rares chez l'homme. Mais l'acide fluorhydrique, comme les fluorures, peuvent causer des intoxications aiguës survenant par absorption ou par voie cutanée.

\subsection{Conclusion}

Allaway a rassemblé dans un tableau les concentrations et les quantités en certains éléments dans les diverses phases de l'environnement. Il en est reproduit un extrait concernant ceux qui ont été étudiés (tab. 67).

\section{Résultats d'enquêtes}

\subsection{Eléments de pollution}

Les teneurs que l'on a retrouvées dans le lait sont largement inférieures à celles indiquées comme toxiques... et lorsqu'on a pu retrouver des traces des éléments cherchés.

\subsection{Oligoéléments}

Durant les deux enquêtes (1973 et 1974-1975) la plus ou moins grande importance de la contamination du lait après la traite a pu être constatée pour le fer, et à un moindre degré pour le cuivre. 
TABLEAU 67

Concentrations et quantités en certains oligoéléments dans diverses phases de l'environnement (d'après Allaway)

\begin{tabular}{|c|c|c|c|c|c|c|c|c|}
\hline Elément & $\begin{array}{l}\text { Concentration } \\
\text { dans les } \\
\text { réserves } \\
\text { géochimiques } \\
\text { (1) }\end{array}$ & $\begin{array}{l}\text { Concentration } \\
\text { dans les sols } \\
\text { (total) } \\
\text { (2) }\end{array}$ & $\begin{array}{c}\text { Concentration } \\
\text { dans les } \\
\text { plantes } \\
\text { (3) }\end{array}$ & $\begin{array}{l}\text { Niveaux cri- } \\
\text { tiqueis dans les } \\
\text { diètes } \\
\text { animales } \\
(4)\end{array}$ & $\begin{array}{l}\text { Concentration } \\
\text { dans le } \\
\text { sang humain } \\
\text { (4) (5) }\end{array}$ & $\begin{array}{c}\text { Extraction } \\
\text { minière } \\
\text { annuelle } \\
\text { (6) }\end{array}$ & $\begin{array}{c}\text { Absorption } \\
\text { annuelle par } \\
\text { les plantes de } \\
\text { prairies et } \\
\text { les culiures } \\
\text { (7) }\end{array}$ & $\begin{array}{c}\text { Temps } \\
\text { d'épuisement } \\
\text { du sol } \\
\text { (7) }\end{array}$ \\
\hline Arsenic & $\begin{aligned} \text { p.p.m. } & \\
a & : 2 \\
b & : 1-3 \\
c & : 0,003\end{aligned}$ & $\begin{array}{l}\text { p.p.m. } \\
6(0,1-40)\end{array}$ & $\begin{array}{l}\text { p.p.m. } \\
1(0,5-5) ; \text { non } \\
\text { exigé. }\end{array}$ & $\begin{array}{l}\text { p.p.m. } \\
\text { Non exigé ; } \\
\text { certains mé- } \\
\text { dicaments ; } \\
\text { As }^{3} \text {, forte to- } \\
\text { xicité ; As } \\
\text { toxicité mo- } \\
\text { dérée. }\end{array}$ & $\begin{array}{c}\mu \mathrm{g} / 100 \mathrm{ml} \\
49\end{array}$ & $\begin{array}{c}\mathrm{kg} \\
2,7 \times 10^{6}\end{array}$ & $\begin{array}{l}\mathrm{kg} \\
10^{7}\end{array}$ & $\begin{array}{c}\text { années } \\
10^{2}\end{array}$ \\
\hline Cadmium & $\begin{array}{l}a: 0,2 \\
b: 0,03-3 \\
c: 0,00011\end{array}$ & $0,06(0,01-7)$ & $\begin{array}{l}(0,2-0,8) ; \text { non } \\
\text { exigé ; toxi- } \\
\text { que. }\end{array}$ & $\begin{array}{l}\text { Non exigé ; } \\
\text { toxicité modé- } \\
\text { rée à forte. }\end{array}$ & $\begin{array}{l}4 \text {; accumu- } \\
\text { lation rénale. }\end{array}$ & $10^{7}$ & $10^{7}$ & $10^{2}$ \\
\hline Cuivre & $\begin{array}{l}a: 55 \\
b: 5-45 \\
c: 0,003\end{array}$ & $20(2-100)$ & $\begin{array}{l}\text { Exigé : } 2-4 ; \\
\text { ten eur nor- } \\
\text { male : } 4-15 ; \\
\text { toxicité au- } \\
\text { dessus de } 20 \text {. }\end{array}$ & $\begin{array}{l}\text { Exigé : } 1-10 ; \\
\text { influence du } \\
\text { Mo ; faible } \\
\text { toxicité. }\end{array}$ & 100 & $4 \times 10^{9}$ & $10^{8}$ & $10^{3}$ \\
\hline Fluor & $\begin{array}{l}a: 625 \\
b: 250-750 \\
c: 1,3\end{array}$ & $200(30-300)$ & $\begin{array}{l}\text { Non exigé; te- } \\
\text { neur norma- } \\
\text { le } 2-20 \text {; toxi- } \\
\text { cité }>50 \text {. }\end{array}$ & $\begin{array}{l}\text { Non exigé ; } \\
\text { utile pour } \\
\text { les os et les } \\
\text { dents ; toxi- } \\
\text { cité modé- } \\
\text { rée. }\end{array}$ & $\begin{array}{l}\text { 36 } \\
1000 \text { p. p. m. } \\
\text { dans le tissu } \\
\text { osseux. }\end{array}$ & $8 \times 10^{8}$ & $10^{s}$ & $10^{4}$ \\
\hline
\end{tabular}


TABLEAU 67 (suite)

Concentrations et quantités en certains oligoéléments dans diverses phases de l'environnement (d'après Allaway)

\begin{tabular}{|c|c|c|c|c|c|c|c|c|}
\hline Elément & $\begin{array}{c}\text { Concentration } \\
\text { dans les } \\
\text { réserves } \\
\text { géochimiques } \\
\text { (1) }\end{array}$ & $\begin{array}{c}\begin{array}{c}\text { Concentration } \\
\text { dans les sols } \\
\text { (total) }\end{array} \\
\text { (2) }\end{array}$ & $\begin{array}{c}\text { Concentration } \\
\text { dans les } \\
\text { plantes } \\
\text { (3) }\end{array}$ & $\begin{array}{l}\text { Niveaux cri- } \\
\text { tiques dans les } \\
\text { dietes } \\
\text { animales } \\
(4)\end{array}$ & $\begin{array}{l}\text { Concentration } \\
\text { dans le } \\
\text { sang humain } \\
\text { (4) (5) }\end{array}$ & $\begin{array}{c}\text { Extraction } \\
\text { minière } \\
\text { annuelle } \\
(6)\end{array}$ & \begin{tabular}{|c|} 
Absorption \\
annuelle par \\
les plantes de \\
prairies et \\
les cultures \\
$(7)$
\end{tabular} & $\begin{array}{c}\text { Temps } \\
\text { d'épuisement } \\
\text { du sol } \\
\text { (7) }\end{array}$ \\
\hline Plomb & 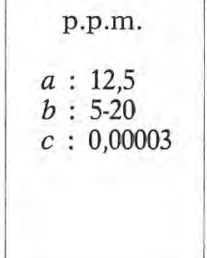 & $\begin{array}{c}\text { p.p.m. } \\
10(2-200)\end{array}$ & $\begin{array}{l}\text { p.p.m. } \\
\text { Non exigé; te- } \\
\text { neur norma- } \\
\text { le : 0,1-10; } \\
\text { toxi que en } \\
\text { solution nu- } \\
\text { tritive. }\end{array}$ & $\begin{array}{l}\text { Non exigé : } \\
\text { toxicité mo- } \\
\text { dérée. }\end{array}$ & $\begin{array}{c}\mu \mathrm{g} / 100 \mathrm{ml} \\
27\end{array}$ & $\begin{array}{c}\mathrm{kg} \\
2 \times 10^{9}\end{array}$ & $\begin{array}{l}\mathrm{kg} \\
10^{7}\end{array}$ & $\begin{array}{c}\text { années } \\
10^{4}\end{array}$ \\
\hline Manganèse & $\begin{array}{l}a: 1000 \\
b: 50-1100 \\
c: 0,002\end{array}$ & $850(100-4000)$ & $\begin{array}{l}\text { Exigé ; teneur } \\
\text { normale: } 15- \\
100 \text {; la toxi- } \\
\text { cité dépend } \\
\text { du rapport } \\
\mathrm{Fe} / \mathrm{Mn} .\end{array}$ & $\begin{array}{l}\text { Exigé : } 10-40 \text { : } \\
\text { faible toxici- } \\
\text { té. }\end{array}$ & 3 & $6 \times 10^{9}$ & $10^{9}$ & $10^{3}$ \\
\hline Zinc & $\begin{array}{l}a: 70 \\
b: 10-100 \\
c: 0,01\end{array}$ & $50(10-300)$ & $\begin{array}{l}\text { Exigé : } 8-15 \\
\text { toxicité } \\
>200 .\end{array}$ & $\begin{array}{l}\text { Exigé : } 10-40 ; \\
\text { faible toxici- } \\
\text { té. }\end{array}$ & 650 & $3 \times 10^{9}$ & $10^{9}$ & $10^{3}$ \\
\hline
\end{tabular}

(1) (2) (6) D'après Bowen H. J. M. $a$ : roches ignées, $b$ : roches sédimentaires, $c$ : eau de mer.

(3) D'après Chapman H. D. Les concentrations sont exprimées par rapport à la matière sèche.

(4) D'après Underwood E. J. et diverses sources. Forte toxicité : une concentration de la diète inférieure à 10 p.p.m. peut être nuisible. Toxicité modérée : une concentration de 10 à 100 p.p.m. peut être nuisible. Faible toxicité : il faut une concentration d'au moins 100 p.p.m. pour observer des effets nuisibles. Toutes les concentrations sont exprimées par rapport à la matière sèche de la diète complète. Le fluor serait très toxique dans l'eau mais faiblement toxique dans les aliments.

(5) D'après Bowen H. J. M.

(7) Méthode d'estimation, voir Allaway W. H. 
TABLEAU 68. - Troupeaux en race pure F.F.P.N.

\begin{tabular}{|c|c|c|c|c|c|c|c|}
\hline \multicolumn{2}{|r|}{ Région } & \multirow{2}{*}{$\begin{array}{c}\text { Hte-Marne } \\
\text { Hte-Saône } \\
\text { Rhône - Loire } \\
\text { Isère - Jura } \\
7\end{array}$} & \multirow{2}{*}{$\begin{array}{c}\text { Yonne } \\
5\end{array}$} & \multirow{2}{*}{$\begin{array}{c}\text { Marne } \\
9\end{array}$} & \multirow{2}{*}{$\begin{array}{c}\text { Nord } \\
\text { Pas-de-Calais } \\
9\end{array}$} & \multirow{2}{*}{$\begin{array}{c}\begin{array}{c}\text { Mayenne } \\
\text { Sarthe } \\
\text { Charentes }\end{array} \\
8\end{array}$} & \multirow{2}{*}{$\begin{array}{c}\begin{array}{c}\text { Aquitaine } \\
\text { Midi- } \\
\text { Pyrénées }\end{array} \\
11\end{array}$} \\
\hline 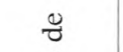 & Troupeaux & & & & & & \\
\hline 屏 & Vaches & 323 & 126 & 346 & 233 & 317 & 314 \\
\hline Z & Vaches par troupeau & 46 & 25 & 38 & 26 & 40 & 29 \\
\hline $\begin{array}{c}\text { Nombre } \\
\text { de }\end{array}$ & Litres par vache & 3660 & 4340 & 4190 & 4300 & 4860 & 3750 \\
\hline \multirow{2}{*}{ 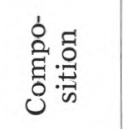 } & Matière grasse $\quad(\mathrm{g} / \mathrm{l})$ & 36,0 & 39,0 & 37,7 & 36,1 & 37,5 & 35,9 \\
\hline & Matière azotée $\quad(\mathrm{g} / \mathrm{l})$ & 31,2 & - & 33,4 & 36,4 & 31,0 & 32,3 \\
\hline \multirow{4}{*}{ 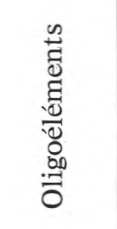 } & $(\mu \mathrm{g} / 1)$ & 999 & 716 & 1497 & 473 & 1510 & 765 \\
\hline & Cuivre & 307 & 97 & 317 & 44 & 92 & 95 \\
\hline & Manganèse $\quad(\mu \mathrm{g} / \mathrm{l})$ & 45 & 57 & 51 & 31 & 59 & 43 \\
\hline & Zinc $\quad(\mu \mathrm{g} / \mathrm{l})$ & 2310 & 2569 & 4415 & 1153 & 3385 & 2526 \\
\hline
\end{tabular}


Les résultats obtenus pour le manganèse et le zinc ont pu être comparés à ceux donnés par la bibliographie.

En ce qui concerne les laits de grand mélange, par exemple, la pauvreté des laits en zinc dans le Sud-Ouest correspond à une aire de carence effectivement démontrée, et d'autant plus que le maïs entre plus dans la ration alimentaire.

Mais ce sont les laits de microrégions qui donnent plus l'idée des phénomènes, que ceux des macrorégions pour lesquels jouent le mélange des origines. Ainsi les observations effeotuées sur laits de troupeaux peuvent être intéressantes.

\subsection{Laits de troupeaux}

Parmi les quatre-vingt-dix-neuf troupeaux ayant participé à l'enquête 1973, la plupart était en race pure. Les tableaux 68 et 69 indiquent les caractéristiques de ces troupeaux ainsi que les résultats concernant les quatre oligoéléments étudiés :

\subsubsection{Troupeaux en race pure F.F.P.N.}

A part un troupeau à effectif très important, les résultats concernant les laits en provenance du Centre-Est ou du Sud-Ouest ont sensiblement les mêmes compositions et les productions moyennes par vache les plus basses.

Les lait du Nord de la France semblent particulièrement pauvres en cuivre, manganèse et zinc.

L'Yonne et les départements de l'Ouest-Sud-Ouest ont une teneur en cuivre semblable (\# $95 \mu \mathrm{g} / 1$ ) ; le Centre-Est et la Marne ayant des teneurs beaucoup plus importantes.

Le Centre-Est et le Sud-Ouest ont des teneurs en manganèse et en zinc semblables. Les laits de la Marne sont moyennement riches en manganèse et très riches en zinc. Les laits de l'Yonne, de la Mayenne, de la Sarthe et des Charentes sont les plus riches en manganèse et moyennement riches en zinc ; ceux de 1'Yonne ayant la même teneur en zinc que ceux du Sud-Ouest.

\subsubsection{Comparaison entre races}

$\mathrm{Si}$ les troupeaux ne sont pas de même importance, ceux de Montbéliardes et de F.F.P.N. d'une part et ceux de Flamandes et de F.F.P.N. d'autre part ont la même quantité de lait livré par vache. Par contre la production des Normandes est nettement inférieure à celle de troupeaux F.F.P.N. particulièrement performants.

Il a été vu dans la bibliographie que la teneur en cuivre et en zinc dépendait de la race et que certaines régions présentaient des carences effectivement démontrées (tab. 64).

On constate effectivement une certaine différence pour le cuivre et le zinc, à tempérer pour le cuivre du fait d'une contamination importante possible après la traite : Centre-Est et Ouest (cuivre) ; Centre-Est et Nord (zinc). 
TABLEAU 69. - Comparaison entre races

\begin{tabular}{|c|c|c|c|c|c|c|c|}
\hline \multicolumn{2}{|r|}{ Région } & \multicolumn{2}{|c|}{$\begin{array}{l}\text { Hte-Marne - Hte Saône } \\
\text { Rhône - Loire } \\
\text { Isère - Jura }\end{array}$} & \multicolumn{2}{|c|}{ Nord - Pas-de-Calais } & \multicolumn{2}{|c|}{ Mayenne - Sarthe - Charentes } \\
\hline & Race & Montbéliarde & F.F.P.N. & Flamande & F.F.P.N. & Normande & F.F.P.N. \\
\hline \multirow{4}{*}{ 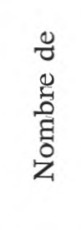 } & Troupeaux & 13 & 7 & 4 & 9 & 7 & 8 \\
\hline & Vaches & 248 & 323 & 158 & 233 & 188 & 317 \\
\hline & Vaches par troupeau & 19 & 46 & 40 & 26 & 27 & 40 \\
\hline & Litres par vache & 3600 & 3660 & 4500 & 4300 & 3500 & 4860 \\
\hline \multirow{2}{*}{ 苞: } & Matière grasse $(\mathrm{g} / \mathrm{l})$ & 34,7 & 36,0 & 39,0 & 36,1 & 41,0 & 37,5 \\
\hline & Matière azotée (g/l) & 32,8 & 31,2 & 36,5 & 36,4 & 32,0 & 31,0 \\
\hline \multirow{4}{*}{ 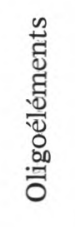 } & $(\mu \mathrm{g} / \mathrm{l})$ & 846 & 999 & 944 & 473 & 1591 & 1510 \\
\hline & Cuivre $\quad(\mu \mathrm{g} / \mathrm{l})$ & 140 & 307 & 48 & 44 & 167 & 92 \\
\hline & Manganèse $\quad(\mu \mathrm{g} / \mathrm{l})$ & 43 & 45 & 32 & 31 & 56 & 59 \\
\hline & Zinc $\quad(\mu \mathrm{gl})$ & 2586 & 2310 & 1350 & 1153 & 3387 & 3385 \\
\hline
\end{tabular}


TABLEAU 70. - Récapitulatif des résultats et des valeurs données par la littérature

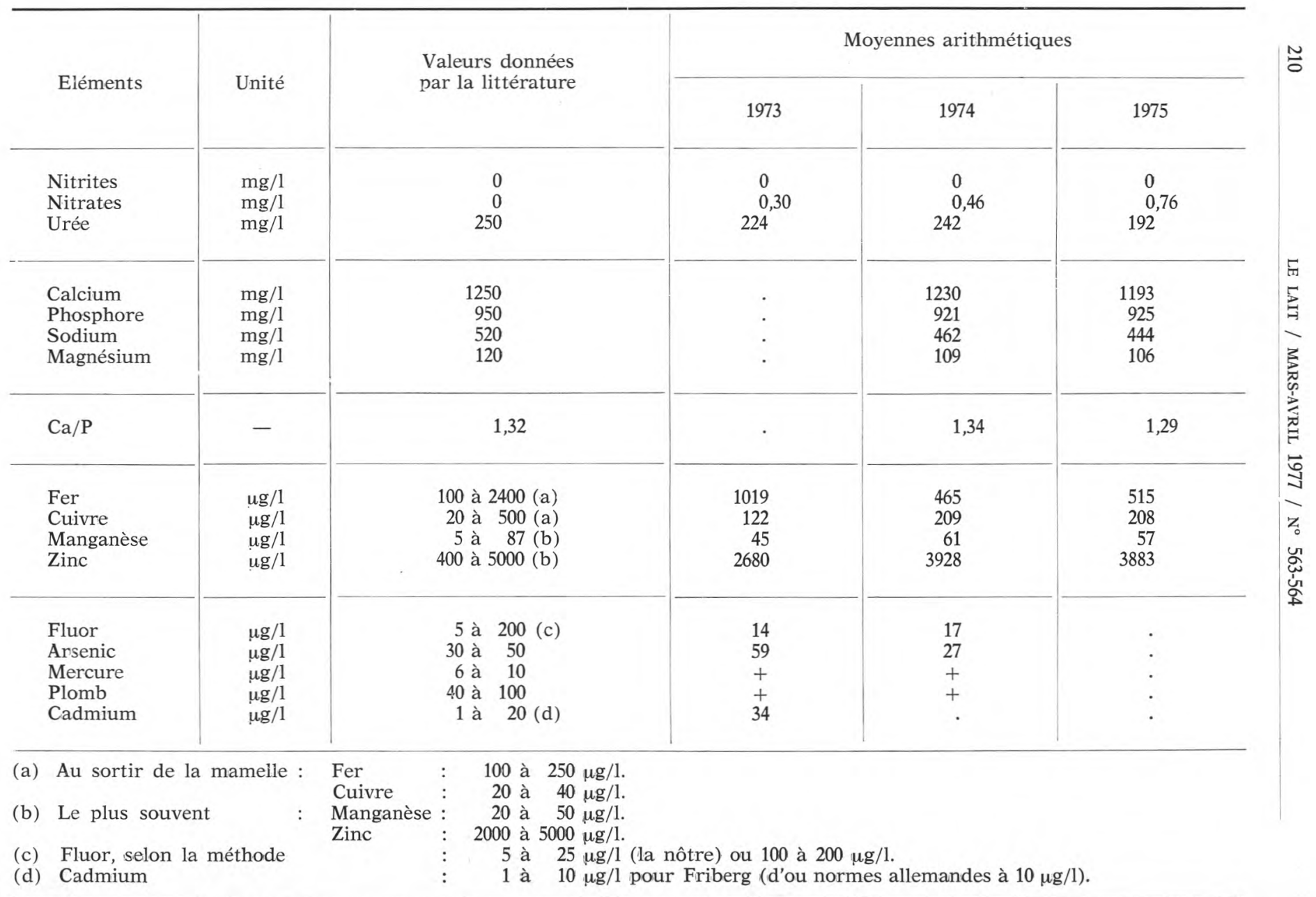


Mais il semble que ces différences entre races sont nettement inférieures aux différences régionales. Le Nord étant particulièrement pauvre en ces oligoéléments ; l'Ouest assez riche et le Centre-Est se situant juste à la moyenne entre les deux autres régions.

\section{Conclusion}

Dans le tableau 70 sont comparées les valeurs données par la littérature et les moyennes arithmétiques de l'ensemble des résultats trouvés (laits individuels et de mélange) durant les années 19731974-1975 :

- Nitrites-nitrates : le lait au sortir de la mamelle n'en contient pas. Il y a donc une contamination du lait après la traite ; contamination qui semble augmenter d'année en année ;

- Urée : la valeur donnée par la littérature semble être un plafond que l'on atteint seulement dans le cas de graves problèmes alimentaires pour le bétail ;

- Calcium, phosphore, sodium, magnésium : les valeurs indiquées dans la littérature ont été rarement atteintes excepté en Normandie et dans l'Ouest. Il semblerait que les teneurs en calcium, sodium et magnésium ont tendance à diminuer alors que les teneurs en phosphore ont une légère tendance à augmenter ; d'où une baisse du rapport $\mathrm{Ca} / \mathrm{P}$ entre 1974 et 1975 ;

- Fer, cuivre : les résultats traduisent une plus ou moins importante contamination du lait après la traite ;

- Manganèse, zinc, fluor : les teneurs du lait en ces trois éléments sont conformes à ce qui a été trouvé dans la littérature ;

- Arsenic : les laits de producteurs sont en moyenne plus contaminés que les laits de grand mélange, bien que faiblement pollués ;

- Mercure, plomb : à part quelques rares échantillons, il n'a été décelé que des traces non analysables de ces deux éléments ;

- Cadmium : si l'on considère les normes allemandes récemment publiées $(10 \mu \mathrm{g} / \mathrm{l})$, l'ensemble des laits de producteurs examiné en 1973 serait non conforme, mais il n'y a pas lieu de s'alarmer.

Pour la plupart des éléments plastiques, des normes de besoins ont été définies par la F.A.O. et l'O.M.S., par tranches d'âges. Ces normes sont retranscrites dans le tableau 71 en regard des valeurs régionales extrêmes constatées et des moyennes arithmétiques nationales calculées durant les années 1973, 1974 et 1975.

Il ressort du tableau 71 que la consommation de 11 de lait par jour apporte suffisamment de cuivre, de calcium et même de zinc. En général la moyenne de consommation du lait liquide est d'environ $1 / 21$; dans ce cas l'apport de calcium est insuffisant pour une femme en état de grossesse et d'allaitement. 
TABLEAU 71. - Besoins nutritionnels de l'homme en éléments plastiques

\begin{tabular}{|c|c|c|c|c|c|c|c|}
\hline \multirow{2}{*}{ Eléments } & \multirow{2}{*}{ Unité } & \multirow{2}{*}{\multicolumn{2}{|c|}{$\begin{array}{c}\text { Besoins nutritionnels } \\
\text { de l'homme } \\
\text { en } \mathrm{mg} / \mathrm{j} \text { ou } \mu \mathrm{g} / \mathrm{j} \\
\text { (O.M.S./F.A.O. 1974) }\end{array}$}} & \multicolumn{3}{|c|}{$\begin{array}{l}\text { Valeurs régionales externes } \\
\text { et moyennes nationales }\end{array}$} & \multirow{2}{*}{$\begin{array}{c}\text { Pourcentage } \\
\text { de couverture } \\
\text { des besoins } \\
\text { pour } 11 \text { de lait }\end{array}$} \\
\hline & & & & 1973 & 1974 & 1975 & \\
\hline Calcium & $\mathrm{mg} / \mathrm{l}$ & $\begin{array}{l}\text { Enfant } \\
\text { Adulte } \\
\text { Grossesse } \\
\text { Allaitement }\end{array}$ & $\begin{array}{l}400 \text { à } 700 \\
400 \text { à } 500 \\
1000 \text { à } \\
1200\end{array}$ & $\cdot$ & $\begin{array}{c}1148 \text { à } 1300 \\
1230\end{array}$ & $\begin{array}{c}1132 \text { à } 1224 \\
1193\end{array}$ & $\begin{array}{r}160 \text { à } 325 \\
225 \text { à } 325 \\
94 \text { à } 130\end{array}$ \\
\hline Phosphore & $\mathrm{mg} / \mathrm{l}$ & - & & . & $\begin{array}{c}845 \text { à } 981 \\
921\end{array}$ & $\begin{array}{c}860 \text { à } 971 \\
925\end{array}$ & - \\
\hline Sodium & $\mathrm{mg} / \mathrm{l}$ & - & & . & $\begin{array}{c}414 \text { à } 507 \\
462\end{array}$ & $\begin{array}{c}411 \text { à } 479 \\
444\end{array}$ & - \\
\hline Magnésium & $\mathrm{mg} / \mathrm{l}$ & 200 à 3 & 300 & . & $\begin{array}{c}102 \text { à } 119 \\
109\end{array}$ & $\begin{array}{l}99 \text { à } 118 \\
106\end{array}$ & 33 à 60 \\
\hline Fer & $\mathrm{mg} / \mathrm{l}$ & $\begin{array}{l}\text { Enfant } \\
\text { Homme } \\
\text { Femme }\end{array}$ & $\begin{array}{r}5 \text { à } 10 \\
5 \text { à } 9 \\
14 \text { à̀ } 28\end{array}$ & $\begin{array}{c}0,584 \text { à } 1,448 \\
1,019\end{array}$ & $\begin{array}{c}0,342 \text { à } 1,054 \\
0,465\end{array}$ & $\begin{array}{c}0,347 \text { à } 0,830 \\
0,515\end{array}$ & 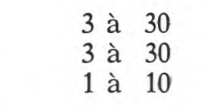 \\
\hline Zinc & $\mathrm{mg} / 1$ & 2,2 à 2 & & $\begin{array}{c}1,217 \text { à } 4,362 \\
2,680\end{array}$ & $\begin{array}{c}3,168 \text { à } 4,757 \\
3,928\end{array}$ & $\begin{array}{c}3,335 \text { à } 4,205 \\
3,883\end{array}$ & 6 à 150 \\
\hline Cuivre & $\mu \mathrm{g} / 1$ & $\begin{array}{l}\text { Adolescent } \\
\text { Adulte }\end{array}$ & $\begin{array}{l}40 \\
30\end{array}$ & $\begin{array}{c}49 \text { à } 330 \\
122\end{array}$ & $\begin{array}{c}132 \text { à } 366 \\
209\end{array}$ & $\begin{array}{c}131 \text { à } 315 \\
208\end{array}$ & $\begin{array}{l}120 \text { à } 900 \\
160 \text { à } 1200\end{array}$ \\
\hline Manganèse & $\mu \mathrm{g} / 1$ & - & & 31 à 63 & $\begin{array}{c}51 \text { à } \\
61\end{array}$ & $\begin{array}{c}50 \text { à } \\
57\end{array}$ & \\
\hline
\end{tabular}

* Selon le régime et la disponibilité du zinc. 
Par contre, les apports en magnésium et surtout en fer, sont particulièrement insuffisants ; surtout pour les femmes, pendant la période de la menstruation.

Les modifications de la composition en éléments du lait constatées au cours de ces enquêtes, nous incite à poursuivre de telles études ; par exemple étude de la variation de la composition :

- sur des laits d'animaux de race pure dans les mêmes régions ou dans des régions différentes ;

- sur des laits provenant de vaches d'une même race appartenant à des exploitations de taille différente par exemple :

plus de cinquante vaches laitières

trente à cinquante

dix à trente

moins de dix ;

- en fonction de certains régimes alimentaires.

Il a semblé que de telles études pourraient être suivies d'une façon beaucoup plus sûre dans un nombre restreint de laiteries appartenant à cinq régions bien définies au cours des années 1973 à 1975 :

$\begin{array}{ll}\text { Est } & \text { Montbéliarde } \\ \text { Nord } & \text { F.F.P.N. } \\ \text { Normandie } & \text { Normande } \\ \text { Ouest } & \text { Normande - F.F.P.N. } \\ \text { Centre } & \text { Divers }\end{array}$

Sur les laits ainsi récoltés pourraient être suivis non seulement la composition en éléments du lait, mais aussi les rapports entre les anions et cations, les équilibres nutritionnels et les équilibres technologiques.

Les enquêtes 1973-1975 ont permis de mettre au point des techniques de prélèvements et des techniques de préparation des échantillons et d'extraction suffisamment fiables pour aborder ces études plus poussées.

Enfin des travaux plus particuliers permettront de préciser certains facteurs comme :

- le rapport pouvant exister entre la qualité de l'eau de boisson et la qualité du lait (présence des nitrates dans le lait) ;

- les déficits en cuivre, zinc et manganèse de certaines régions.

La voie ouverte par les enquêtes est fonction des nombreux travaux des recherches fondamentales et appliquées qu'elles soient faites à l'I.N.R.A., au C.N.R.S., au C.E.A., dans les universités ou ailleurs. Cette voie permet aux différents chercheurs de voir l'accomplissement possible de leurs travaux sur le terrain et aussi de voir remonter vers eux d'autres problèmes à résoudre donc de nouveaux sujets de recherches. 


\section{Remerciements}

Nous tenons à remercier :

- Pour la documentation, la bibliographie et les éléments de recherches fournis, ainsi que pour l'aide apportée dans les analyses et la rédaction des résumés bibliographiques :
M. Bittel
C.E.A. Fontenay-aux-Roses,
Mme Erb
professeur d'hydrologie et d'hygiène de l'eau à la Faculté de Pharmacie de Lille.
$\begin{array}{ll}\text { M. } & \text { Guéguen } \\ \text { M. } & \text { Lamand } \\ \text { M. } & \text { Raoul }\end{array}$
I.N.R.A.-C.N.R.Z., Jouy-en-Josas.
I.N.R.A.-C.R.Z.V., Theix.
M. Rémond I.N.R.A.-C.R.Z.V., Theix.

- Les laiteries qui ont participé aux enquêtes :

O.R.L.A.C.* ; Coopérative Laitière de Crest ; Société Nouvelles des Fromageries Girod ; Comité Technique du Comté* ; S.A. Bongrain Gérard* ; Fromageries Bel* ; Fromageries F. Paul Renard* ; Lorraine-Lait ; Fromageries Lincet* ; Fromageries Loevenbruck ; Prospérité Fermière* ; La Roche aux Fées ; Coopérative Laitière de la Thiérache; La C.L.A.R.A. ; La C.L.H.N. ; Gervais-Danone; Union Laitière Normande ; La Sapiem-Préval ; Coopérative Laitière de Ploudaniel ; Union Laitière Bretonne ; La Co La Ré Na ; La C.A.N.A.* ; Coopérative Laitière de Sèvre et Belle ; Coopérative Laitière de Lezay ; Union Laitière Pyrénéenne* ; Tempé Lait ; S.L.M. et F.G.T. Réunies à Rodez* ; Laiteries Richesmonts ; Centre-Lait ; S.A. Laiteries Hubert Triballat ; Coopérative Laitière de la Vallée du Nahon.

- Les producteurs qui ont accepté de répondre au questionnaire et fourni du lait.

* Laiteries ou groupes de laiteries ayant participé à l'enquête 1973 chez les producteurs.

\section{Bibliographie}

GuÉGUeN (L.) (1971). - La composition minérale du lait et son adaptation aux besoins minéraux du jeune. Ann. Nutr. Alim., 25, A 335 - A 381.

JouRNÉES SCIENTIFIQUES DU C.N.E.R.N.A. - Oligoéléments et nutrition. 15 au 18 mars 1971. Compte rendu du C.N.R.S.

Dequidt (J.), ERb (F.), Brice (A.) et Gromez-Potencier (J.) (1974). - Détermination d'éléments minéraux. Trace dans le lait. Ann. Fals. Exp. Chim., nov.-déc., 67, $\mathrm{n}^{\circ}$ 723-724, 567-576.

Roux (M.), Luquet (F. M.) et CASALIS (J.) (1974). - Sur la teneur en sels minéraux de laits de vache des régions du Nord et du Sud-Ouest de la France. Ann. Fals, Exp. Chim., nov.-déc., 67, n 723-724, 577-583.

REMOND (B.) (1975). - La teneur du lait de vache en nitrate. Le Lait, tome LV, $\mathrm{n}^{\circ}$ 547, juillet-août, 390-395.

Mouillet (L.), Luouet (F. M.) et Casalis (J.). - Contribution à l'étude des variations de la teneur en sels minéraux du lait de vache dans différentes régions françaises.

Bittel (R.). - Composition minérale des laits en France. Synthèse non publiée.

Groupe A.L.F. (MARION). - Réponse à l'enquête F.I.L. sur la pollution minérale des laits et produits laitiers français. 